\title{
Fabrication of Microscale Carbon Nanotube Fibers
}

\author{
Gengzhi Sun, Yani Zhang, and Lianxi Zheng \\ School of Mechanical and Aerospace Engineering, Nanyang Technological University, Singapore 639798 \\ Correspondence should be addressed to Lianxi Zheng, lxzheng@ntu.edu.sg
}

Received 13 October 2011; Accepted 16 December 2011

Academic Editor: Teng Li

Copyright (C) 2012 Gengzhi Sun et al. This is an open access article distributed under the Creative Commons Attribution License, which permits unrestricted use, distribution, and reproduction in any medium, provided the original work is properly cited.

\begin{abstract}
Carbon nanotubes (CNTs) have excellent mechanical, chemical, and electronic properties, but realizing these excellences in practical applications needs to assemble individual CNTs into larger-scale products. Recently, CNT fibers demonstrate the potential of retaining CNT's superior properties at macroscale level. High-performance CNT fibers have been widely obtained by several fabrication approaches. Here in this paper, we review several key spinning techniques including surfactant-based coagulation spinning, liquid-crystal-based solution spinning, spinning from vertical-aligned CNT arrays, and spinning from CNT aerogel. The method, principle, limitations, and recent progress of each technique have been addressed, and the fiber properties and their dependences on spinning parameters are also discussed.
\end{abstract}

\section{Introduction}

Carbon nanotubes (CNTs) are the stiffest (Yong's modulus) and strongest (yield strength) materials are yet measured. Their tensile strength is about $11-63 \mathrm{GPa}$ for individual multiwalled CNTs (MWNTs) and 13-52 GPa for individual single-walled CNTs (SWNTs) [1-3]. They are also good conductors for electricity and heat [4-8]. These extraordinary properties make them attractive for advanced applications. In order to fully utilize their superior properties at practical scale, CNTs need to be prepared into larger size assemblies, such as microscale CNT fibers. Recent progresses [9-12] in neat CNT fibers demonstrate the possibility of retaining CNT's excellent properties at larger-scale and more practicable level. The CNT fibers have been reported to have tensile strength of $1 \sim 3 \mathrm{GPa}$, Young's modulus of $100 \sim 260 \mathrm{GPa}$, toughness of $100 \sim 900 \mathrm{~J} \cdot \mathrm{g}^{-1}$, and density of $0.2 \mathrm{~g} \cdot \mathrm{cm}^{-3}$ [9]. These progresses motivate further study of lightweight and high-strength composites for possible structural applications.

Numerous methods have been developed to assemble such fibers [13]. Generally, these techniques could be divided into two groups: solution-spinning methods [14-18] and solid-spinning techniques. In solution-based spinning, CNTs need to be dispersed into a liquid first, and then spun into fibers, by a process similar to that used for polymeric fibers.
In solid-spinning processes, CNT fibers could be spun from vertically aligned CNT arrays $[19,20]$, cotton-like CNT mats $[21,22]$, or from an aerogel of CNTs formed in chemical vapor deposition (CVD) reaction zone $[23,24]$. The performances of CNT fibers are strongly dependent on processing methods and the detail process parameters. Here in this paper, we will review several key spinning techniques and their recent progress.

\section{Solution-Based Spinning}

CNT fibers could be produced by using "solution-spinning" method, just like most synthetic fibers created from a concentrated viscous liquid. These processes consist of dispersing the CNTs in solution and then recondensing them in a stream of another solution, which serves as the coagulant. The first critical challenge in development of this method is the difficulty of processing CNTs in liquid state. CNTs are inert in pristine state and tend to bundle together due to the strong van der Waals interactions, making them difficult to disperse uniformly in aqueous or any organic solvents. Some methods have been utilized to overcome this problem through oxidation and grafting with different functionalities [25-29], but these methods normally destroy CNT's intrinsic structures and properties. Thus, they are not favored for fiber spinning. Shaffer and Windle [30] have previously suggested 
that CNTs can be viewed as analogous to high-aspect ratio and rigid-rod polymers. According to this analog, CNTs are supposed to be applicable to two types of solution-based spinning methods: coagulation spinning and liquid-crystal solution spinning.

2.1. Surfactant-Based Coagulation Spinning. Generally, the principle of the "coagulation spinning" used for synthetic fiber processing could be depicted as when a polymer solution is extruded through a thin capillary tube and injected into a bath that contains a second liquid in which the solvent is soluble but the polymer is not, the polymer will condense and form a fiber due to the phase separation. Employing this "coagulation spinning" method for CNT fiber fabrication, the CNTs need to be dispersed into a liquid solution at an almost molecule level so that they could be manipulated and aligned well. Surfactants are widely utilized for this purpose because of their ability to absorb/wrap at the surface of individual CNTs and prevent them from rebundling. This spinning approach was initially adopted by Vigolo et al. [14]. In their fabrication process, as shown schematically in Figure 1, arc-discharge-produced SWNTs were firstly dispersed in an aqueous solution by using sodium dodecyl sulfate (SDS) as surfactant, then injected into a rotating bath of aqueous polyvinyl alcohol (PVA) solution, which serves as the coagulant. During this process, PVA displaced the surfactant, causing CNTs collapse and forming ribbon-like elastomeric gel-fibers. These fragile fibers were pulled from the coagulation bath at a rate of about $0.01 \mathrm{~m} \cdot \mathrm{min}^{-1}$ in order to form solid fibers. Such fibers were washed by immersing in successive water container in order to remove excess PVA and surfactant residues and then were dried by pulling them out of water bath.

It is found that one critical parameter to obtain a good dispersion of CNTs is the amount of SDS. When the concentration of SDS is too low, large and dense clusters of the CNTs will still exist in solution even after sonication, which means that the amount of surfactant is too low to produce an efficient coating and induce enough electrostatic repulsion that could counterbalance van der Waals attractions. On the other hand, when the concentration of SDS is too high, the osmotic pressure of the excess micelles causes depletion-induced aggregation. They found that an optically homogeneous solution could be formed with $0.35 \mathrm{wt} \%$ CNTs and $1 \mathrm{wt} \%$ SDS for CNTs with particular diameter and length. In addition, flow-induced alignment could lead to a preferential orientation of the CNTs in fibers $[14,31]$ and has a close relationship to relative flow rate between injection solution and coagulant solution, as shown in Figure 2. The coagulant must flow faster than the gel-fiber in order to stretch the fiber along the axis direction and promote alignment of CNTs in the fiber. This could be accomplished by rotating the coagulant container.

This coagulation-based fiber spinning technique is exciting because of its simplicity and ability to produce fibers with very high CNT loadings (60 wt. \%). The final CNT/PVA composite fibers exhibited a tensile strength in the order of $0.1 \mathrm{GPa}$ and a Young's modulus varying between 9 and $15 \mathrm{GPa}$. In contrast to most ordinary carbon fibers, CNT

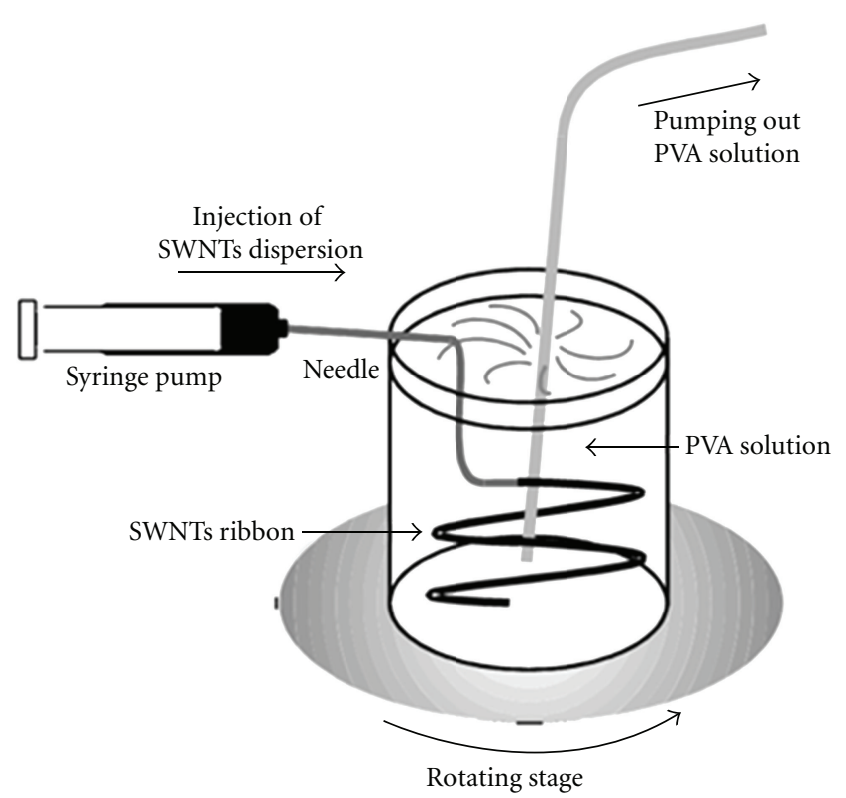

FIGURE 1: Schematic of the experimental setup used to make SWNT ribbons. The capillary tip was orientated so that the SWNT injection was tangential to the circular trajectory of the polymer solution [14].

fibers (shown in Figure 3) can be heavily bent and even tightly tied without breaking.

The main challenges existing in this method include dispersion of SWNTs at high concentrations, low processing rate, and the poor fiber performance. In order to improve the mechanical performance of as-spun CNT fibers, various modified methods have been developed. By drying CNT fibers under load, improved mechanical properties were obtained with a tensile strength of $230 \mathrm{MPa}$ and a Young's modulus of $45 \mathrm{GPa}[32,33]$. By hot drawing the fibers, Miaudet et al. [34] drew such PVA fibers at elevated temperatures, and the fibers yield a strength of $1.8 \mathrm{GPa}$, a modulus of $45 \mathrm{GPa}$, and a toughness of $55 \mathrm{~J} \cdot \mathrm{g}^{-1}$ at $11 \%$ strain. Dalton et al. [35] further advanced the spinning apparatus to spin fibers continuously by injecting CNT dispersion into a cylinder with the coagulant flowing in the same direction. They were able to spin a reel of CNT gel fibers and then converted it into 100 meters solid CNT-composite fibers, at a rate of more than $0.70 \mathrm{~m} \cdot \mathrm{min}^{-1}$. The final fibers exhibit an increased mechanical performance with a tensile strength of $1.8 \mathrm{GPa}$ and a Young's modulus of up to $80 \mathrm{GPa}$. Coagulation spinning has also been done with solutions other than PVA. For example, Lynam et al. [36] produced CNT biofibers based on a wet-spinning process in which biomolecules acted as both the dispersant and coagulant. These fibers possessed strength of $0.17 \mathrm{GPa}$ and modulus of $0.146 \mathrm{GPa}$.

Since the existence of the second component polymer will add complicity of processing and this second component is usually an insulator, which will compromise the conductive property of as-spun fibers, pure CNT fibers are favorable in some circumstances. Kozlov et al. [17] developed a polymer-free solution spinning method. Pure CNT 


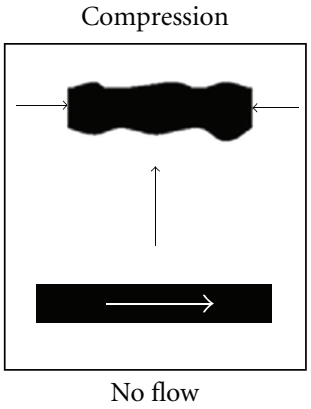

(a)

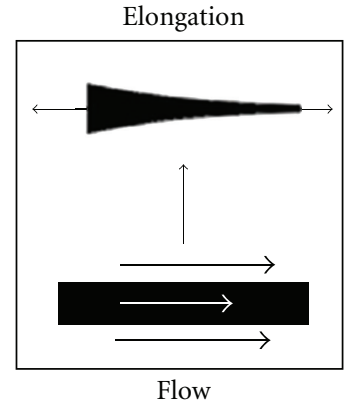

(b)

FIGURE 2: (a) When the coagulation bath does not flow or flows slower than injecting rate, a net compressive force acts on the gel-like fiber, compromising the alignment. (b) When the coagulant flows along the extruded fiber and faster than the injecting rate, a net stretching force will be resulted to increase the alignment [14].
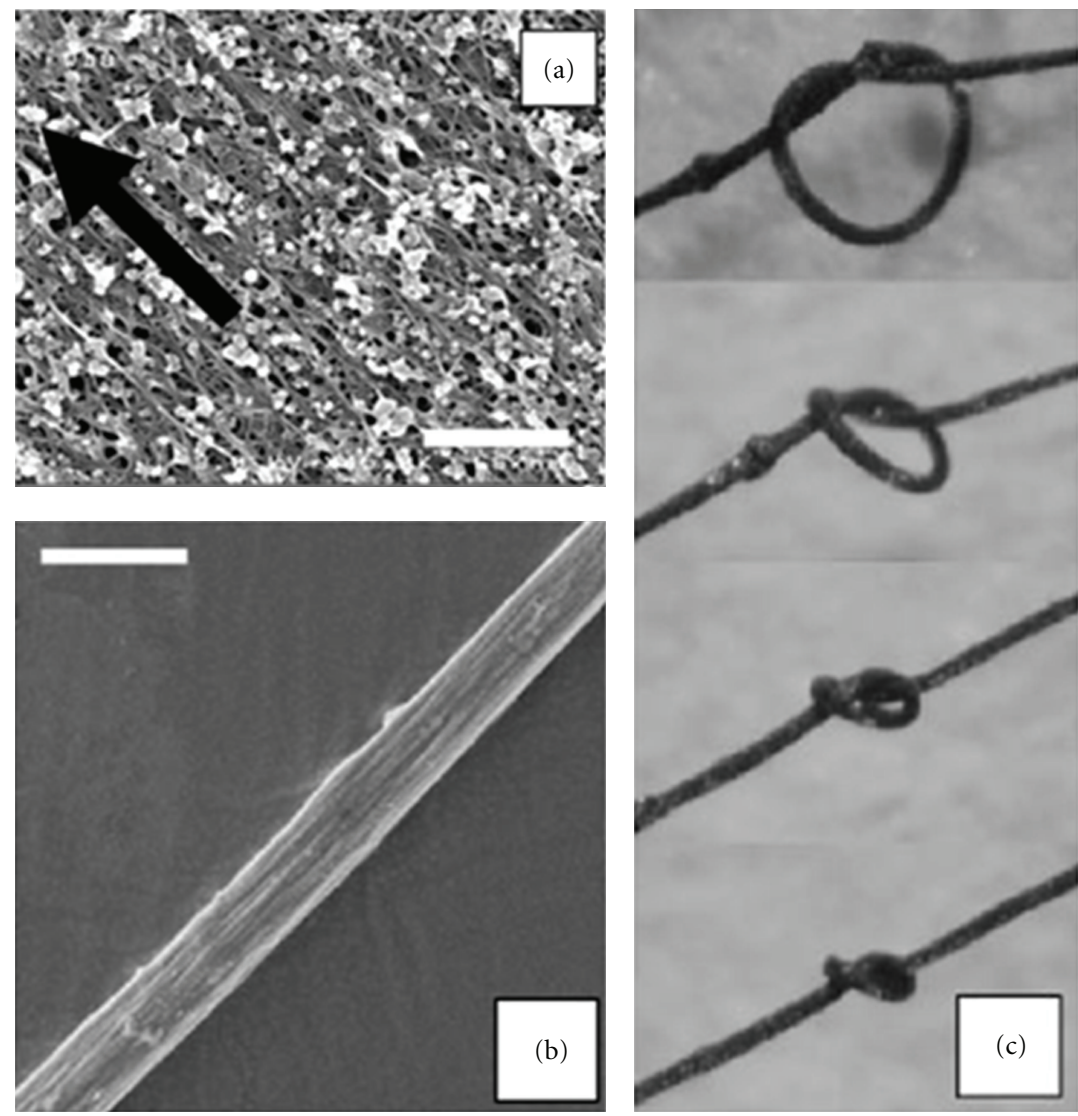

Figure 3: (a) A dry ribbon deposited on a glass substrate. (The black arrow indicates the main axis of the ribbon, which corresponds to the direction of the initial fluid velocity.) (b) A CNT fiber. (c) Knots reveal the high flexibility and resistance to torsion of the CNT microfibers. Scale bars: $500 \mathrm{~nm}$ and $25 \mu \mathrm{m}$ for (a) and (b) [14].

fibers can be produced from CNT/surfactant/water solutions. However, the mechanical properties of the as-spun fibers are not impressive, showing a specific strength of $65 \mathrm{MPa} \cdot \mathrm{g}^{-1} \cdot \mathrm{cm}^{-3}$, specific modulus of $12 \mathrm{GPa} \cdot \mathrm{g}^{-1} \cdot \mathrm{cm}^{-3}$, and electrical conductivity of $140 \mathrm{~S} \cdot \mathrm{cm}^{-1}$.

2.2. Liquid Crystal-Based Solution Spinning. Spinning from lyotropic liquid-crystalline solution of rigid-rod molecules is another important method used for fiber production. As shown in Figure 4, CNTs are similar to high-aspect ratio and rigid-rod polymers and exhibit liquid crystallinity feature $[37,38]$. Ericson et al. [16] first successfully produced wellaligned macroscopic fibers composed solely of SWNTs from lyotropic solutions in super acids. Fuming sulfuric acid charges SWNTs and promotes them to order into an aligned phase with individual mobile CNTs surrounded by acid 

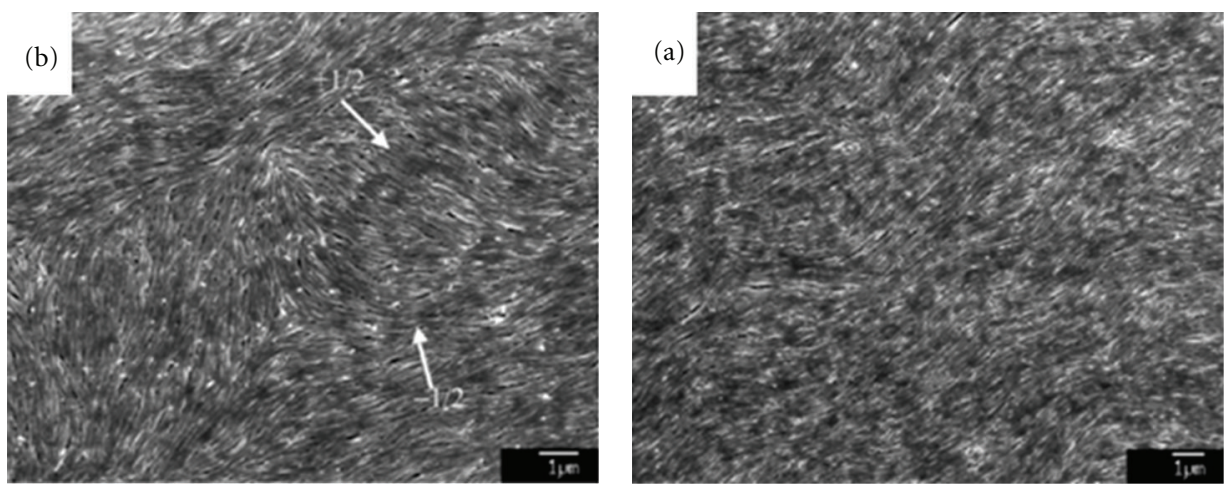

FIGURE 4: Scanning electron microscopy (SEM) images of a dried MWNT film. (a) The director fields around a pair of disclinations of topological strength $+1 / 2$ and $-1 / 2$ and (b) the region toward the edge of the film which is free of disclinations [37, 38].

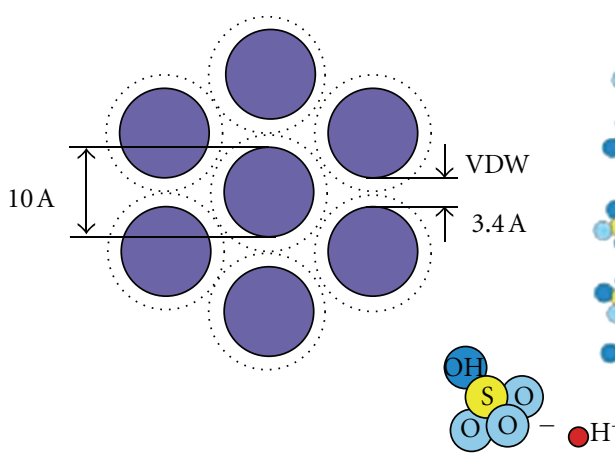

(a)

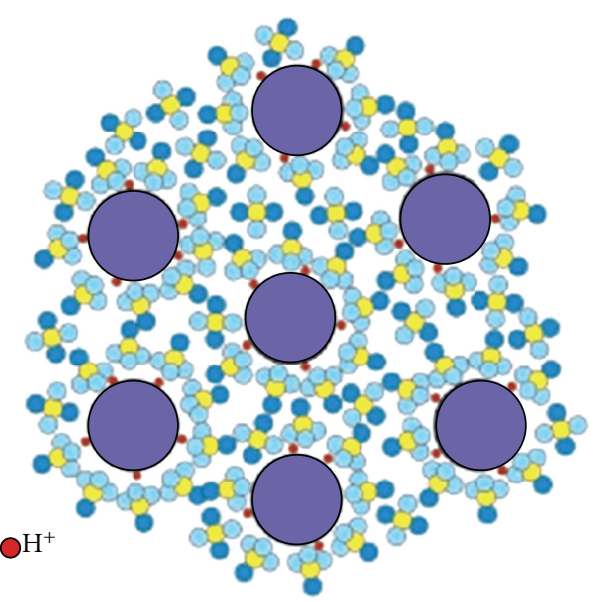

(b)

FIGURE 5: A model illustrating the swelling of SWNT ropes in sulfuric acid. (a) A cartoon of SWNTs in van der Waals contact within a neat fiber. (b) The same SWNT fiber after reexposure to sulfuric acid [16].

anions. This ordered dispersion was then extruded into a coagulant bath (either diethyl ether, 5\% sulfuric acid, or water) to form continuous macroscopic CNT fibers.

The possible mechanism, that high concentration CNTs could be dispersed in superacid ( $100+\%$ sulfuric acid), is the repulsive interaction between CNTs generated in superacids due to the formation of charge-transfer complexes: individual positively charged CNTs surrounded by a finite number of sulfuric acid anions. At very low concentration, such charged tube-anion complexes behave as Brownian rods. At higher concentration, as shown in Figure 5, the CNTs coalesce and form ordered domains, behaving similarly to nematic liquid crystalline.

The CNT fibers spun by such a process have interesting structural and physical properties, including high orientation, good electrical, and thermal conductivities, and reasonable mechanical properties. The alignment of CNTs within these fibers is within $\pm 15.5^{\circ}$. The strength is $116 \pm$ $10 \mathrm{MPa}$, and the Young's modulus approaches to $120 \pm$ $10 \mathrm{GPa}$. However, some protonation of the material occurs because of prolonged contact with the sulfuric acid. The $\mathrm{CNT} /$ acid system is very sensitive to water; the introduction of even minimal moisture causes phase separation and precipitation of discrete needle-like crystal solvates. And superacid route is also found not effective for MWNTs. To address the last problem, Zhang et al. [39] developed a new coagulation process, by which they spun MWNTs from a liquid-crystalline ethylene glycol dispersion. The MWNT fibers have a Young's modulus of $69 \pm 41 \mathrm{GPa}$ and a yield strain of $0.3 \%$. Fracture occurs typically at strains below $3 \%$ and stresses of $0.15 \pm 0.06 \mathrm{GPa}$.

\section{Solid Spinning}

3.1. Spinning Fibers from Vertical-Aligned CNT Arrays. In order to eliminate the dispersion problem existing in solutionbased spinning methods, spinning CNT fibers directly from as-grown CNT materials seems to be a more convenient way. A breakthrough was made by Jiang et al. [19] in 2002 by simply drawing a neat CNT yarn from a vertically superaligned 

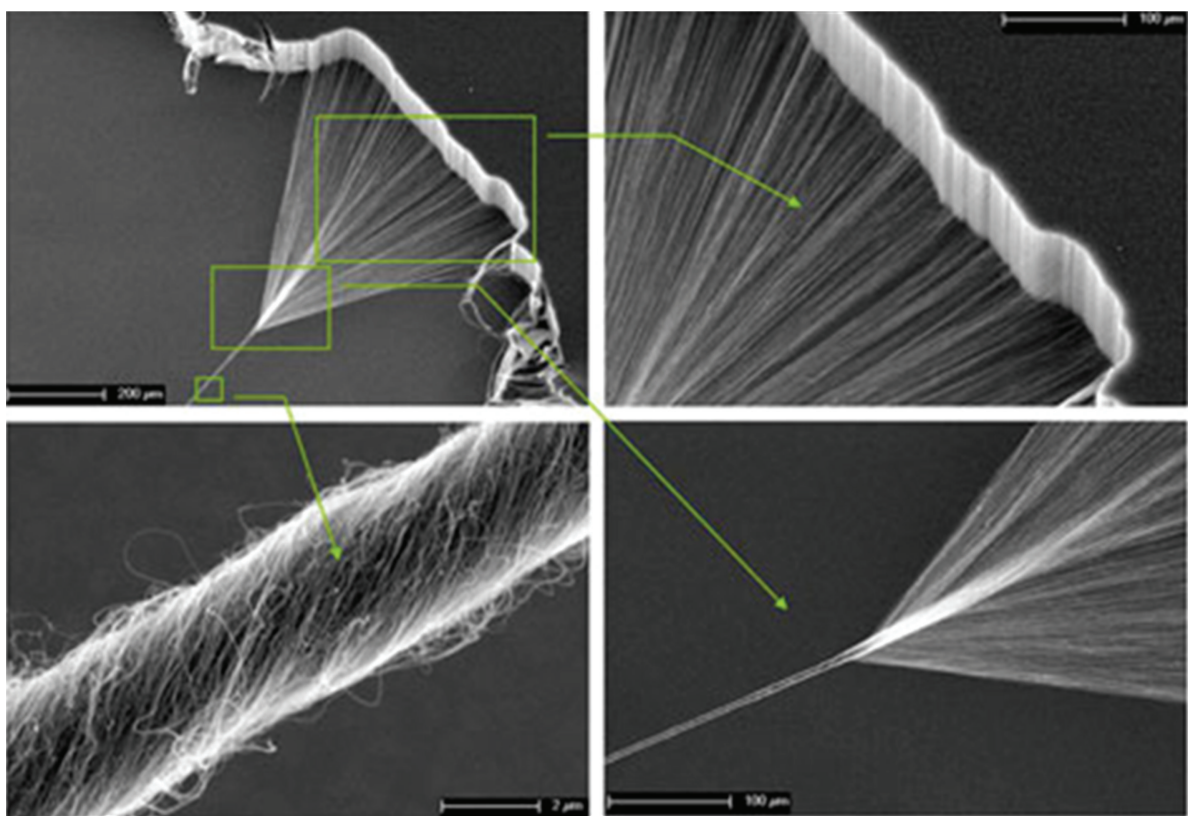

FIgURE 6: SEM images showing the structures formed during the draw-twist process [20].

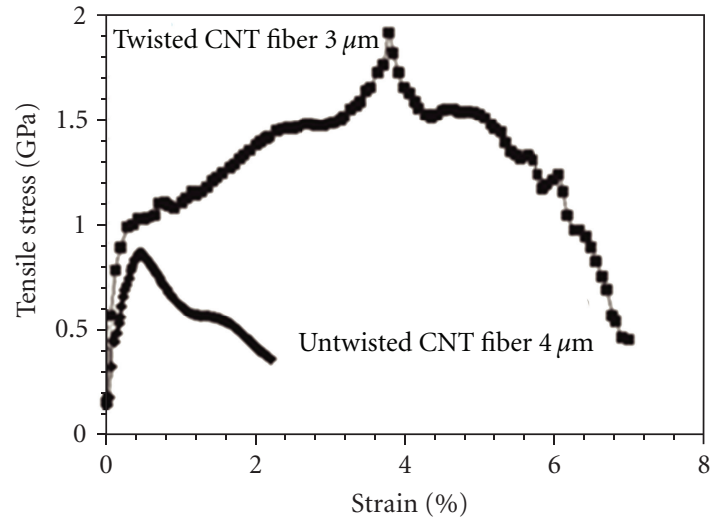

(a)

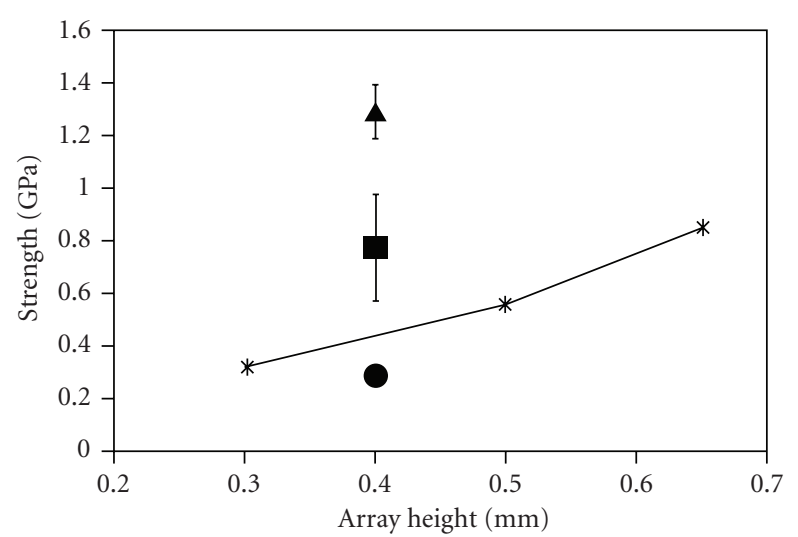

(b)

Figure 7: (a) As-spun and posttwisted small-diameter CNT fibers spun form a $650 \mu \mathrm{m}$ array. (b) A comparison of fiber strengths at different array heights. The black line shows that the strength of CNT fibers increases with the array height, and marks shows the strength dependence on array morphology (the dot represents the data from oxygen-assisted growth that shows poor CNT alignment, the square represents the data from the normal growth, and the triangle represents the data from the hydrogen-assisted growth that shows good CNT alignment) $[10,12]$.

CNT array. They found that CNTs could be self-assembled into yarns of up to $30 \mathrm{~cm}$ in length. Following that, Zhang et al. [40] produced highly orientated, free-standing CNT transparent sheets using a similar method, and further assemble CNTs into fibers by using a draw-twisting spin method [20]. The typical SEM images of fiber spinning processes are shown in Figure 6.

Many applications of these fibers were proposed and demonstrated [41-44], and different postspinning methods were developed to improve their performances. Jiang et al. [19] found that the strength and conductivity of their yarns could be improved after being heated at high temperatures. By introducing twist during spinning to make multiple, torque-stabilized yarns, Zhang et al. [20] achieved yarn strength greater than $460 \mathrm{MPa}$. They emphasized that the load could be transferred effectively between CNTs because of the twisting. In a twisted fiber, individual CNTs are inclined at an angle $\alpha$ with respect to the fiber axis, generating transverse forces which lock the fibers together as a coherent structure. They also found these twisted yarns deformed hysterically over large strain ranges from $0.5 \%$ to $8 \%$, providing up to $48 \%$ energy damping. These yarns could also retain their strength and flexibility even after being heated in air at $450^{\circ} \mathrm{C}$ for an hour or being immersed in liquid nitrogen. In addition to using postspinning treatment, Zhang et al. [9-11] found that mechanical properties can 


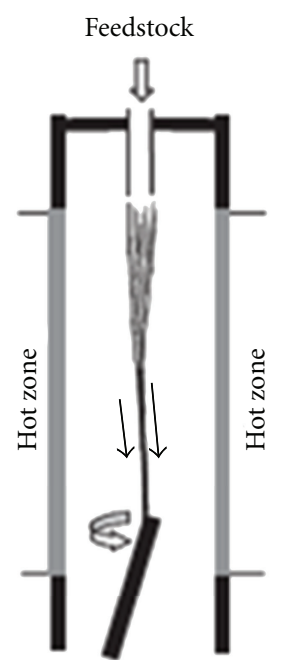

Wind-up

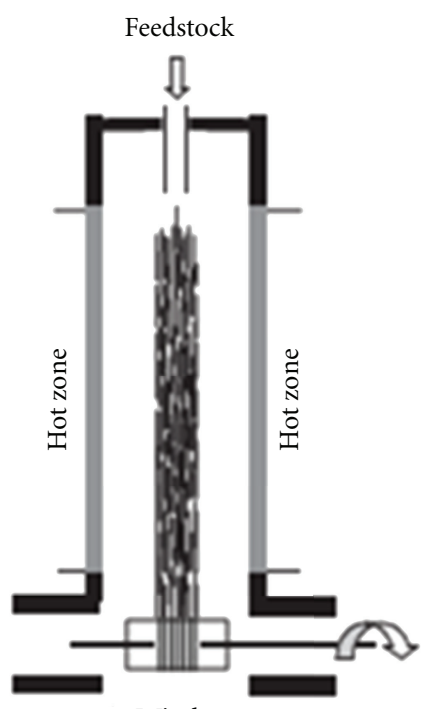

Wind-up
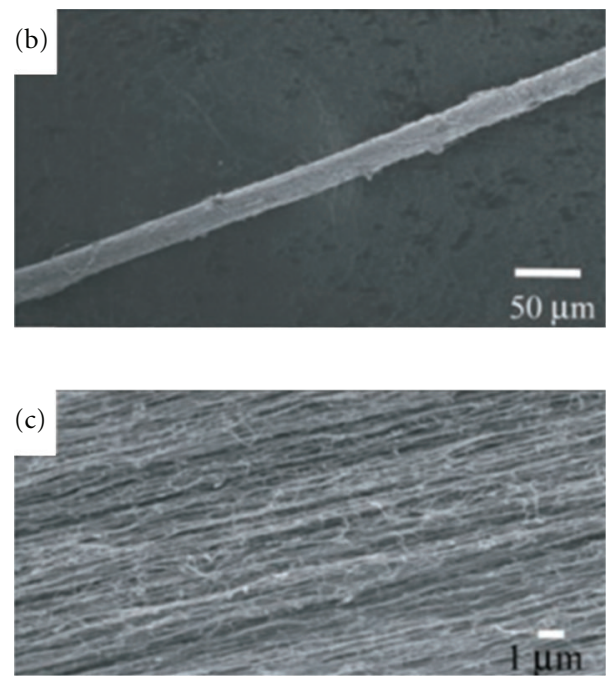

(a)

Figure 8: (a) Schematic of the direct spinning process. The liquid feedstock, in which small quantities of ferrocene and thiophene are dissolved, is mixed with hydrogen and injected into the hot zone, where an aerogel of CNTs form. This aerogel is captured and wound out of the hot zone continuously as a fiber or film. (b) SEM image of a fiber. (c) Well-aligned MWNTs within the fiber [23].
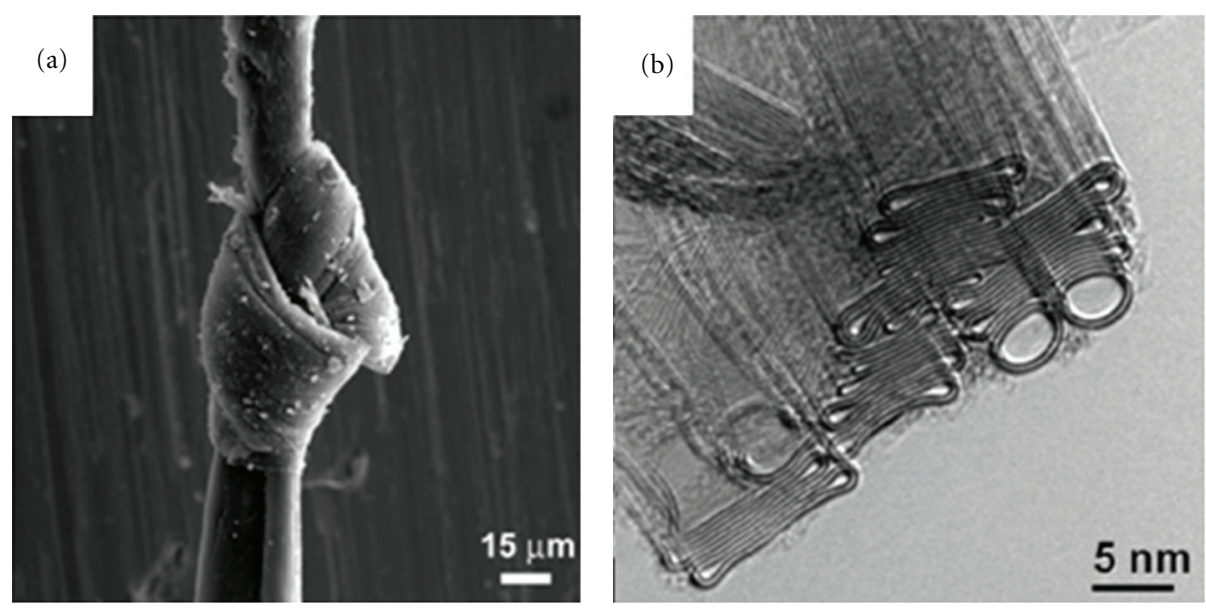

FIGURE 9: Structure of the fiber product. (a) SEM image of knotted fiber. (b) High-resolution transmission electron microscopy (HR-TEM) image of a bundle close to a fiber fracture revealing that the bundles consist, predominantly, of collapsed double-wall nanotubes greater than $5 \mathrm{~nm}[59]$.

be significantly improved by using longer CNT arrays. The tensile strength and stiffness of their fibers spun from a $1 \mathrm{~mm}$ long CNT array were measured in the range of $1.35-3.3 \mathrm{GPa}$ and $100-263 \mathrm{GPa}$, respectively, which are many times stronger and stiffer per weight than the best existing engineering fibers and CNT fibers reported previously. It is obvious that the strength of CNT fibers increased with increasing CNT array length which yields a much larger friction between CNTs. Longer CNTs will also introduce fewer mechanical defects (like the ends of CNTs) per unit fiber length [45-48]. Other factors like structure, purity, density, alignment, and the straightness of CNTs [49] have all been investigated. For instance, in order to get dense packed CNT fibers, surface-tension-driven densification [50, 51] was employed during fiber spinning. Zhang et al. found that after the CNT yarn was pulled through droplets of ethanol, the several centimeters wide yarn shrank into a tight fiber typically $20-30 \mu \mathrm{m}$ in diameter and the strength of the CNT yarn was dramatically improved. The CNT alignment is found especially crucial for fiber properties $[52,53]$ and could be measured by Raman and X-ray diffraction [54, 55]. Zheng et al. [12] have observed a strong correlation between the array morphologies (the straightness of CNTs) and the fiber properties: well-aligned arrays yield 


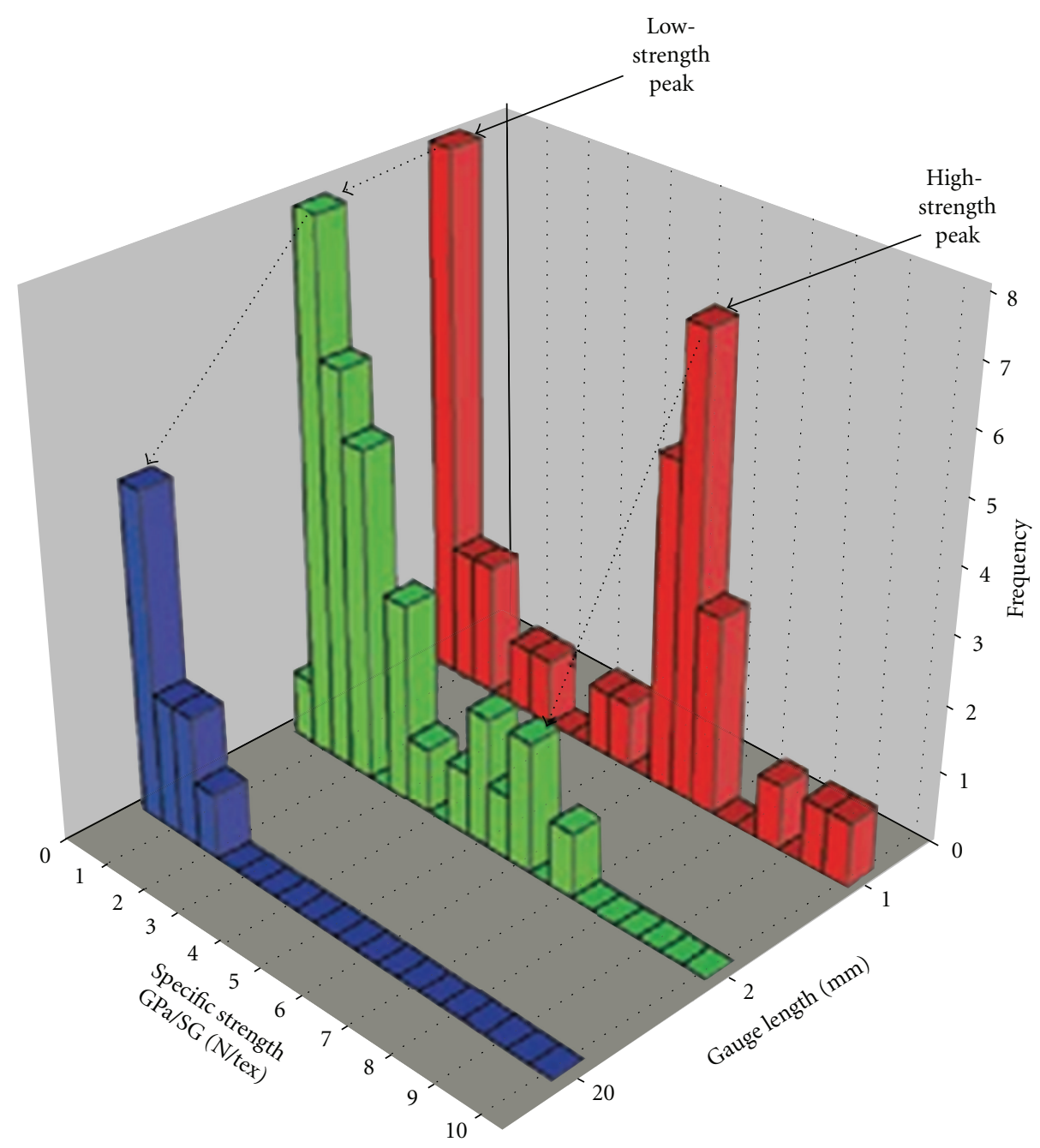

Figure 10: specific strength distribution of CNT fibers at different gauge lengths [24].

high performance, while wavy arrays give poor performance. Figure 7 summarizes the influence of several parameters on fibers' mechanical performances.

Since CNTs in a yarn are nearly parallel-aligned, the CNT yarn is intrinsically an anisotropic material and has a special axis along the drawing direction, which demonstrates many fascinating properties and applications. However, some key issues need to be solved in advance to realize their practical applications. Currently the growth of CNT arrays is easy, but not all CNT arrays could be spun into yarns or fibers. Zhang et al. [50] found that strong van der Waals interactions exist between individual CNTs within superaligned arrays, and this van der Waals force makes the CNTs join end to end, thus forming a continuous yarn during pulling. Meanwhile, Zhang et al. [20] claimed that the formation of yarn was due to the disordered regions at the top and bottom of the CNT arrays, which entangled together forming a loop. Further investigation is needed to understand the underlying spinning mechanism.

3.2. Spinning Fiber from Aerogel of CNTs. Zhu et al. [56] have first reported the formation of a $20 \mathrm{~cm}$ long CNT thread after the pyrolysis of hexane, ferrocene, and thiophene. This work shows the possibility of fiber formation directly in a furnace. Based on this phenomenon, a totally different fiber spinning method was developed by Li et al. [23]. They were able to spin neat CNT fibers directly from an aerogel of CNTs formed in CVD reaction zone, as shown in Figure 8. The precursor materials include liquid hydrocarbon feedstock, ferrocene which forms the iron nanoparticles that act as nucleation sites for the growth of CNTs, and thiophene which is an established rate enhancer for vapor grown carbon fibers [57]. The key requirements for continuous spinning are the formation of CNT aerogel and removal of the product from reaction zone. These were realized through the appropriate choice of reactants, control of the reaction conditions, and continuous withdrawal of the products with a rotating spindle used in various geometries.

Recently, systematic studies of this method have been carried out $[58,59]$. From the view of reactants and growth conditions, the continuous spinning process is possible with a range of oxygen-containing carbon sources. Aromatic hydrocarbons lead to the deposition of carbon particles and thick fibers, but cannot enable a continuous spinning process 
unless they are mixed with another oxygen-containing source. Thiophene is found to be a necessary additive. It was used as an established rate enhancer for vapor growth of carbon fibers [57], but its actual role played in CNT aerogel generation is still open to discussion. However, it is well accepted that sulfur, another additive, plays a major role in promoting carbon-hydrocarbon reactions, especially when associated with iron [57]. Through carefully controlling the growth conditions, the length and diameter of CNT in aerogel could be tuned, and it is found that lower concentrations of iron lead to a greater proportion of SWNTs and double-walled nanotubes (DWNTs) which are favorable for high-performance fibers. For example, it is found that the large diameter DWNTs may collapse within fibers, leading to an increase in friction between individual CNTs, which is beneficial for mechanical performance of CNT fibers [60]. Regarding the processing parameters, it is found that CNT alignment, the density, and microstructures of fibers can be controlled by drawing/winding rate and postprocessing methods. The degree of alignment could be manipulated by adjusting the winding rate as there is a tension introduced into this winding process, which supply a force to align CNTs in the fiber. By introducing the wetting and evaporation of volatile organic liquids such as acetone, the condensation of the CNTs in fibers is greatly increased. Motta et al. [59] have found that the improvement in mechanical strength relates to a unique aspect of fiber microstructure with "dog-bone" shape (shown in Figure 9). They have also shown that the mechanical properties of the fibers directly relate to the type of CNTs, which in turn, can be controlled by the careful adjustment of process parameters.

Through the optimization process, Koziol et al. [24] have found that, by drawing the aerogel at a winding rate of $20 \mathrm{~m} \mathrm{~min}^{-1}$, the strength of the fiber, mainly containing DWNTs, can reach around $10 \mathrm{GPa}$, which is the highest value reported so far. As can be seen in Figure 10, it shows the distribution of specific fiber strengths for a range of gauge lengths (the measure length of the sample). The strength of CNT fibers peaks at around $1 \mathrm{GPa}$ in the case of $20 \mathrm{~mm}$ gauge lengths. As the gauge length decreases, the strength distribution becomes bimodal with a second peak at $6.5 \mathrm{GPa}$, which indicates that the distance between "weak points" along the fibers is on the same order as the gauge length. These "weak points" exist inside the fibers without real interlock between individual CNTs, leading to a decrease in mechanical strength when the fiber is long.

\section{Summary}

In last few years, the development of CNT fibers has shown a sign that superior properties of individual CNTs could be retained at practical size level. A variety of fiber-spinning techniques has been developed, and many posttreatments are utilized to improve the fiber's mechanical properties. Nevertheless, the fiber's performance is still poor compared with individual CNTs or small CNT bundles. Future research need to focus on the understanding of the failure mechanism of CNT fibers, aiming at finding key limiting factors and thus providing reliable and high performance CNT fibers for practical applications.

\section{Acknowledgment}

The authors would like to thank the financial support from Singapore DSTA/DIRP Research Grant (no. POD0814218).

\section{References}

[1] M. F. Yu, O. Lourie, M. J. Dyer, K. Moloni, T. F. Kelly, and R. S. Ruoff, "Strength and breaking mechanism of multiwalled carbon nanotubes under tensile load," Science, vol. 287, no. 5453, pp. 637-640, 2000.

[2] M. F. Yu, B. S. Files, S. Arepalli, and R. S. Ruoff, "Tensile loading of ropes of single wall carbon nanotubes and their mechanical properties," Physical Review Letters, vol. 84, no. 24, pp. 5552-5555, 2000.

[3] B. G. Demczyk, Y. M. Wang, J. Cumings et al., "Direct mechanical measurement of the tensile strength and elastic modulus of multiwalled carbon nanotubes," Materials Science and Engineering A, vol. 334, no. 1-2, pp. 173-178, 2002.

[4] P. Nikolaev, H. Dai, P. Petit, H. Dai, P. Petit et al., "Crystalline ropes of metallic carbon nanotubes," Science, vol. 273, no. 5274, pp. 483-487, 1996.

[5] S. Frank, P. Poncharal, Z. L. Wang, and W. A. de Heer, "Carbon nanotube quantum resistors," Science, vol. 280, no. 5370, pp. 1744-1746, 1998.

[6] J. Hone, M. Whitney, and A. Zettl, "Thermal conductivity of single-walled carbon nanotubes," Synthetic Metals, vol. 103, no. 1-3, pp. 2498-2499, 1999.

[7] S. Berber, Y. K. Kwon, and D. Tománek, "Unusually high thermal conductivity of carbon nanotubes," Physical Review Letters, vol. 84, no. 20, pp. 4613-4616, 2000.

[8] Q. W. Li, Y. Li, X. F. Zhang et al., "Structure-dependent electrical properties of carbon nanotube fibers," Advanced Materials, vol. 19, no. 20, pp. 3358-3363, 2007.

[9] X. F. Zhang, Q. W. Li, T. G. Holesinger et al., "Ultrastrong, stiff, and lightweight carbon-nanotube fibers," Advanced Materials, vol. 19, no. 23, pp. 4198-4201, 2007.

[10] X. F. Zhang, Q. W. Li, Y. Tu et al., "Strong carbon-nanotube fibers spun from long carbon-nanotube arrays," Small, vol. 3, no. 2, pp. 244-248, 2007.

[11] Q. W. Li, X. F. Zhang, R. F. DePaula et al., "Sustained growth of ultralong carbon nanotube arrays for fiber spinning," Advanced Materials, vol. 18, no. 23, pp. 3160-3163, 2006.

[12] L. X. Zheng, G. Z. Sun, and Z. Y. Zhan, "Tuning array morphology for high-strength carbon-nanotube fibers," Small, vol. 6, no. 1, pp. 132-137, 2010.

[13] N. Behabtu, M. J. Green, and M. Pasquali, "Carbon nanotubebased neat fibers," Nano Today, vol. 3, no. 5-6, pp. 24-34, 2008.

[14] B. Vigolo, A. Penicaud, C. Coulon et al., "Macroscopic fibers and ribbons of oriented carbon nanotubes," Science, vol. 290, no. 5495, pp. 1331-1334, 2000.

[15] A. B. Dalton, S. Collins, J. Razal et al., "Continuous carbon nanotube composite fibers: properties, potential applications, and problems," Journal of Materials Chemistry, vol. 14, no. 1, pp. 1-3, 2004.

[16] L. M. Ericson, H. Fan, H. Q. Peng et al., "Macroscopic, neat, single-walled carbon nanotube fibers," Science, vol. 305, no. 5689, pp. 1447-1450, 2004.

[17] M. E. Kozlov, R. C. Capps, W. M. Sampson et al., "Spinning solid and hollow polymer-free carbon nanotube fibers," Advanced Materials, vol. 17, no. 5, pp. 614-617, 2005. 
[18] W. Néri, M. Maugey, P. Miaudet, A. Derré, C. Zakri, and P. Poulin, "Surfactant-free spinning of composite carbon nanotube fibers," Macromolecular Rapid Communications, vol. 27, no. 13, pp. 1035-1038, 2006.

[19] K. L. Jiang, Q. Q. Li, and S. S. Fan, “Nanotechnology: spinning continuous carbon nanotube yarnsyarns-carbon nanotubes weave their way into a range of imaginative macroscopic applications," Nature, vol. 419, p. 801, 2002.

[20] M. Zhang, K. R. Atkinson, and R. H. Baughman, "Multifunctional carbon nanotube yarns by downsizing an ancient technology," Science, vol. 306, no. 5700, pp. 1358-1361, 2004.

[21] L. X. Zheng, X. F. Zhang, Q. W. Li et al., "Carbon-nanotube cotton for large-scale fibers," Advanced Materials, vol. 19, no. 18, pp. 2567-2570, 2007.

[22] L. Ci, N. Punbusayakul, J. Wei, R. Vajtai, S. Talapatra, and P. M. Ajayan, "Multifunctional macroarchitectures of doublewalled carbon nanotube fibers," Advanced Materials, vol. 19, no. 13, pp. 1719-1723, 2007.

[23] Y. L. Li, I. A. Kinloch, and A. H. Windle, "Direct spinning of carbon nanotube fibers from chemical vapor deposition synthesis," Science, vol. 304, no. 5668, pp. 276-278, 2004.

[24] K. Koziol, J. Vilatela, A. Moisala et al., "High-performance carbon nanotube fiber," Science, vol. 318, no. 5858, pp. 1892 $1895,2007$.

[25] G. Z. Sun, S. W. Liu, K. F. Hua, X. Y. Lv, L. Huang, and Y. J. Wang, "Electrochemical chlorine sensor with multiwalled carbon nanotubes as electrocatalysts," Electrochemistry Communications, vol. 9, no. 9, pp. 2436-2440, 2007.

[26] Y. N. Zhang and L. X. Zheng, "Towards chirality-pure carbon nanotubes," Nanoscale, vol. 2, no. 10, pp. 1919-1929, 2010.

[27] J. B. Xu, K. F. Hua, G. Z. Sun, X. Y. Lv, and Y. J. Wang, "Electrooxidation of methanol on carbon nanotubes supported PtFe alloy electrode," Electrochemistry Communications, vol. 8, no. 6, pp. 982-986, 2006.

[28] P. C. P. Watts, W. K. Hsu, H. W. Kroto, and D. R. M. Walton, "Are bulk defective carbon nanotubes less electrically conducting?” Nano Letters, vol. 3, no. 4, pp. 549-553, 2003.

[29] A. Star, Y. Liu, K. Grant et al., "Noncovalent side-wall functionalization of single-walled carbon nanotubes," Macromolecules, vol. 36, no. 3, pp. 553-560, 2003.

[30] M. S. P. Shaffer and A. H. Windle, "Analogies between polymer solutions and carbon nanotube dispersions," Macromolecules, vol. 32, no. 20, pp. 6864-6866, 1999.

[31] C. Mercader, A. Lucas, A. Derré et al., "Kinetics of fiber solidification," Proceedings of the National Academy of Sciences of the United States of America, vol. 107, no. 43, pp. 1833118335, 2010.

[32] B. Vigolo, P. Poulin, M. Lucas, P. Launois, and P. Bernier, "Improved structure and properties of single-wall carbon nanotube spun fibers," Applied Physics Letters, vol. 81, no. 7, pp. 1210-1212, 2002.

[33] E. Munoz, A. B. Dalton, S. Collins et al., "Multifunctional carbon nanotube composite fibers," Advanced Engineering Materials, vol. 6, no. 10, pp. 801-804, 2004.

[34] P. Miaudet, S. Badaire, M. Maugey et al., "Hot-drawing of single and multiwall carbon nanotube fibers for high toughness and alignment," Nano Letters, vol. 5, no. 11, pp. 2212-2215, 2005.

[35] A. B. Dalton, S. Collins, E. Munoz et al., "Super-tough carbonnanotube fibres - these extraordinary composite fibres can be woven into electronic textiles," Nature, vol. 423, no. 6941, p. 703, 2003.

[36] C. Lynam, S. E. Moulton, and G. G. Wallace, "Carbonnanotube biofibers," Advanced Materials, vol. 19, no. 9, pp. 1244-1248, 2007.
[37] W. H. Song and A. H. Windle, "Isotropic-nematic phase transition of dispersions of multiwall carbon nanotubes," Macromolecules, vol. 38, no. 14, pp. 6181-6188, 2005.

[38] W. Song, I. A. Kinloch, and A. H. Windle, "Nematic liquid crystallinity of multiwall carbon nanotubes," Science, vol. 302, no. 5649, p. 1363, 2003.

[39] S. J. Zhang, K. K. K. Koziol, I. A. Kinloch, and A. H. Windle, "Macroscopic fibers of well-aligned carbon nanotubes by wet spinning," Small, vol. 4, no. 8, pp. 1217-1222, 2008.

[40] M. Zhang, S. L. Fang, A. A. Zakhidov et al., "Strong, transparent, multifunctional, carbon nanotube sheets," Science, vol. 309, no. 5738, pp. 1215-1219, 2005.

[41] L. Xiao, Z. Chen, C. Feng et al., "Flexible, stretchable, transparent carbon nanotube thin film loudspeakers," Nano Letters, vol. 8, no. 12, pp. 4539-4545, 2008.

[42] C. Feng, K. Liu, J. S. Wu et al., "Flexible, stretchable, transparent conducting films made from superaligned carbon nanotubes," Advanced Functional Materials, vol. 20, no. 6, pp. 885-891, 2010.

[43] Y. H. Sun, K. Liu, J. Miao et al., "Highly sensitive surfaceenhanced raman scattering substrate made from superaligned carbon nanotubes," Nano Letters, vol. 10, no. 5, pp. 1747-1753, 2010.

[44] G. Z. Sun, J. Y. Zhou, F. Yu, Y. N. Zhang, J. H. L. Pang, and L. X. Zheng, "Electrochemical capacitive properties of CNT fibers spun from vertically aligned CNT arrays," Journal of Solid State Electrochemistry. In press.

[45] L. X. Zheng, M. J. O'Connell, S. K. Doorn et al., "Ultra-long single-wall carbon nanotubes," Nature Materials, vol. 3, no. 10, pp. 673-676, 2004.

[46] Z. Y. Zhan, Y. N. Zhang, G. Z. Sun, L. X. Zheng, and K. Liao, "The effects of catalyst treatment on fast growth of millimeterlong multi-walled carbon nanotube arrays," Applied Surface Science, vol. 257, no. 17, pp. 7704-7708, 2011.

[47] L. Zheng, B. C. Satishkumar, P. Q. Gao, and Q. Zhang, "Kinetics studies of ultra-long single-walled carbon nanotubes," Journal of Physical Chemistry C, vol. 113, no. 25, pp. 1089610900, 2009.

[48] S. K. Doorn, L. X. Zheng, M. J. O’Connell, Y. T. Zhu, S. M. Huang, and J. Liu, "Raman spectroscopy and imaging of ultralong carbon nanotubes," Journal of Physical Chemistry B, vol. 109, no. 9, pp. 3751-3758, 2005.

[49] J. J. Jia, J. N. Zhao, G. Xu et al., "A comparison of the mechanical properties of fibers spun from different carbon nanotubes," Carbon, vol. 49, no. 4, pp. 1333-1339, 2011.

[50] X. B. Zhang, K. L. Jiang, C. Feng et al., "Spinning and processing continuous yarns from 4 -inch wafer scale super-aligned carbon nanotube arrays," Advanced Materials, vol. 18, no. 12, pp. 1505-1510, 2006.

[51] Y. Wei, K. L. Jiang, X. F. Feng et al., "Comparative studies of multiwalled carbon nanotube sheets before and after shrinking," Physical Review B, vol. 76, no. 4, Article ID 045423, 7 pages, 2007.

[52] V. K. Rangari, M. Yousuf, S. Jeelani, M. X. Pulikkathara, and V. N. Khabashesku, "Alignment of carbon nanotubes and reinforcing effects in nylon-6 polymer composite fibers," Nanotechnology, vol. 19, no. 24, Article ID 245703, 2008.

[53] L. J. Lanticse, Y. Tanabe, K. Matsui et al., "Shear-induced preferential alignment of carbon nanotubes resulted in anisotropic electrical conductivity of polymer composites," Carbon, vol. 44, no. 14, pp. 3078-3086, 2006.

[54] H. H. Gommans, J. W. Alldredge, H. Tashiro, J. Park, J. Magnuson, and A. G. Rinzler, "Fibers of aligned single-walled 
carbon nanotubes: polarized Raman spectroscopy," Journal of Applied Physics, vol. 88, no. 5, pp. 2509-2514, 2000.

[55] V. Pichot, M. Burghammer, S. Badaire et al., "X-ray microdiffraction study of single-walled carbon nanotube alignment across a fibre," Europhysics Letters, vol. 79, no. 4, Article ID 46002, 2007.

[56] H. W. Zhu, C. L. Xu, D. H. Wu, B. Q. Wei et al., "Direct synthesis of long single-walled carbon nanotube strands," Science, vol. 296, no. 5569, pp. 884-886, 2002.

[57] G. G. Tibbetts, C. A. Bernardo, D. W. Gorkiewicz, and R. L. Alig, "Role of sulfur in the production of carbon fibers in the vapor phase," Carbon, vol. 32, no. 4, pp. 569-576, 1994.

[58] M. Motta, Y. L. Li, I. Kinloch, and A. Windle, "Mechanical properties of continuously spun fibers of carbon nanotubes," Nano Letters, vol. 5, no. 8, pp. 1529-1533, 2005.

[59] M. Motta, A. Moisala, I. A. Kinloch, and A. H. Windle, "High performance fibres from "dog bone" carbon nanotubes," Advanced Materials, vol. 19, no. 21, pp. 3721-3726, 2007.

[60] X. H. Zhang and Q. W. Li, "Enhancement of friction between carbon nanotubes: an efficient strategy to strengthen fibers," ACS Nano, vol. 4, no. 1, pp. 312-316, 2010. 

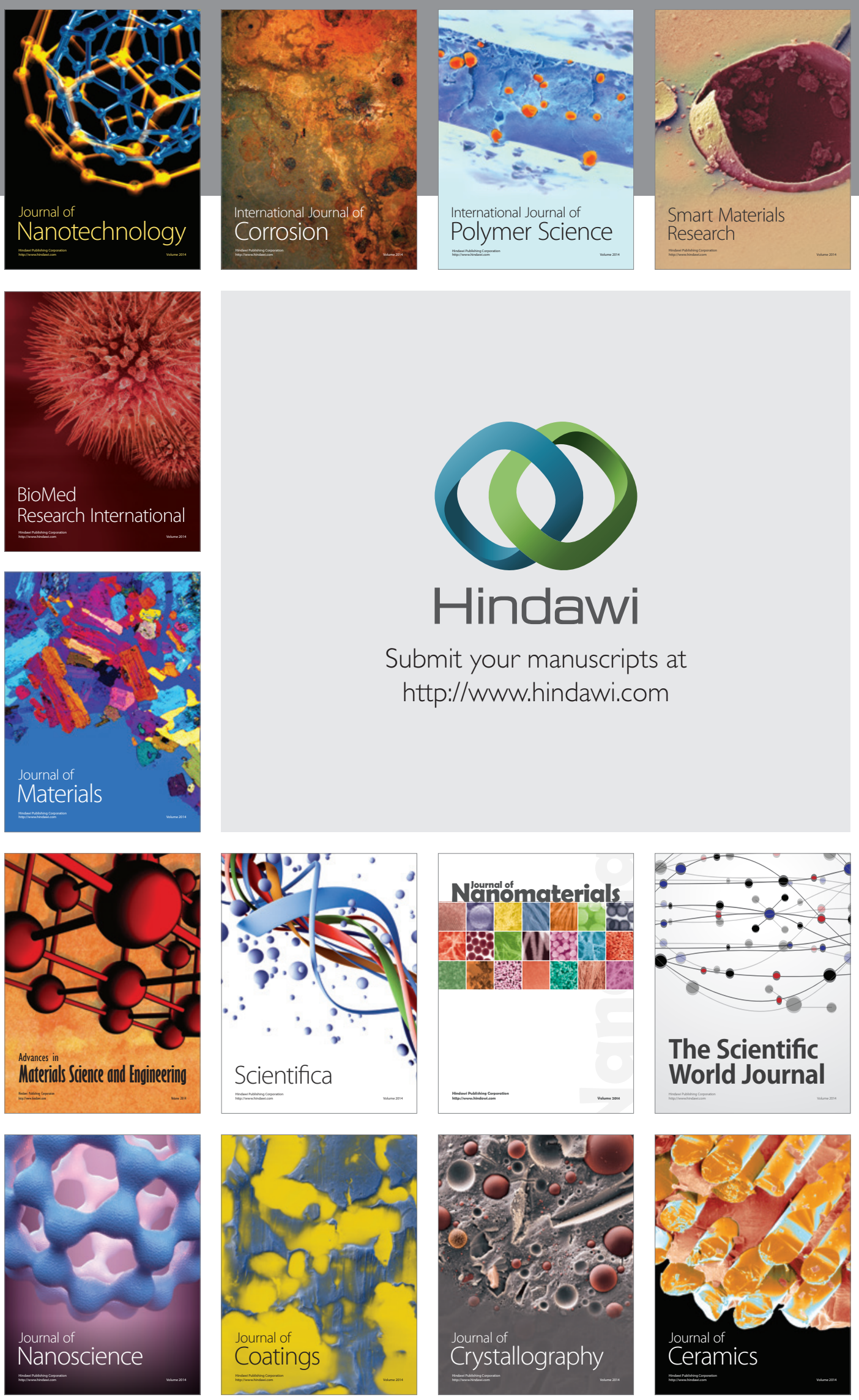

The Scientific World Journal

Submit your manuscripts at

http://www.hindawi.com

\section{World Journal}

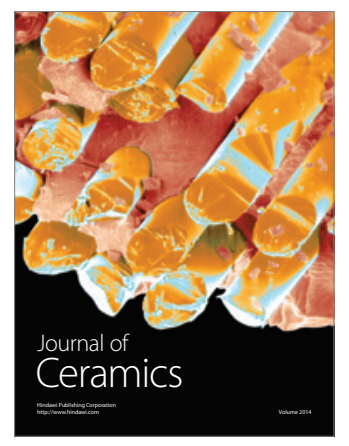

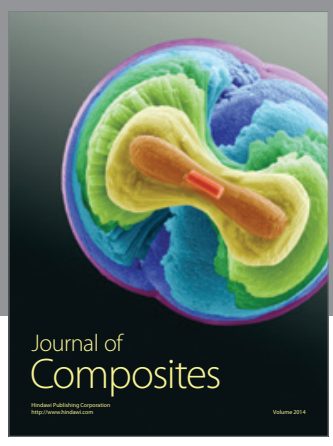
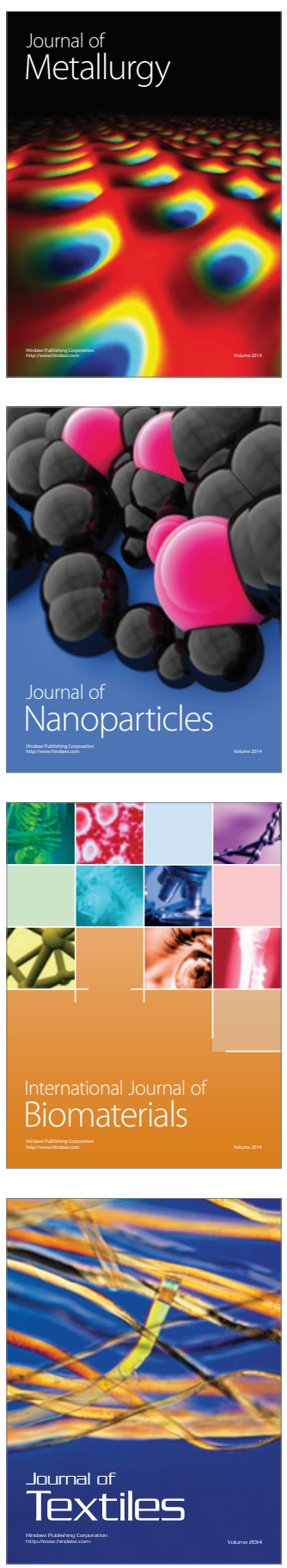